\title{
Mapping and Genome Sequence Analysis of Chromosome 5 Regions Involved in Bladder Cancer Progression
}

\author{
Andrzej Kram, Li Li, Ruo Dan Zhang, Dong Sup Yoon, Jay Y Ro, Dennis Johnston, \\ Herbert Barton Grossman, Steven Scherer, and Bogdan Czerniak
}

Department of Pathology (AK, LL, RDZ, DSY, JYR, BC), Department of Biomathematics (DJ), and Department of Urology (HBG), The University of Texas M. D. Anderson Cancer Center, Houston; and Department of Molecular and Human Genetics (SS), Human Genome Sequencing Center, Baylor College of Medicine, Houston, Texas

\begin{abstract}
SUMMARY: We studied the evolution of allelic losses on chromosome 5 by whole-organ histologic and genetic mapping in 234 mucosal DNA samples of 5 cystectomy specimens with invasive bladder cancer and preneoplastic changes in adjacent urothelium. The frequency of alterations in individual loci was verified on 32 tumors and 29 voided urine samples from patients with bladder cancer. Finally, deleted regions on chromosome 5 were integrated with the human genome contigs and sequence-based databases. Deleted regions on chromosome 5 involved in intraurothelial phases of bladder neoplasia defined by their nearest flanking markers and predicted size were identified as follows: q13.3-q22 (D5S424-D5S656; 38.8 centimorgan [CM]); q22-q31.1 (D5S656-D5S808; 19.2 cM), q31.1-q32 (D5S816-SPARC; 11.5 cM), and q34 (GABRA1-D5S415; 6.4 cM). The two most frequently deleted neighbor markers (D5S2055 and D5S818) mapping to q22-q31.1 defined a $9 \mathrm{cM}$ region, which may contain genes that play an important role in early phases of urinary bladder carcinogenesis. Human genome database analysis provided an accurate map of deleted regions with positions of 138 known genes and revealed several smaller gene-rich areas representing putative targets for further mapping. The strategic approach presented here, which combines whole-organ histologic and genetic mapping with analysis of the rapidly emerging human genome sequence database, facilitates identification of genes potentially involved in early phases of bladder carcinogenesis. (Lab Invest 2001, 81:1039-1048).
\end{abstract}

$C$ ancer develops via multiple, cumulative steps, many of which precede the development of clinically and even microscopically recognizable disease. Mapping and human genome sequence analysis of chromosomal regions involved in clinically occult preinvasive phases of neoplasia may provide valuable clues for more specific studies of early events in human carcinogenesis and could lead to the development of novel early detection markers as well as preventive strategies.

We have previously reported the identification of several putative tumor suppressor gene loci involved in early preinvasive phases of human urinary bladder carcinogenesis (Chaturvedi et al, 1997; Czerniak et al, 1999, 2000). Bladder tumors were used as a common model of human cancer, which develops by progression of microscopically recognizable in situ precursor conditions, and are easily accessible by various minimally invasive or noninvasive techniques (Gazdar and Czerniak, 2001; Greenlee et al, 2000). The entire

Received April 9, 2001.

This work was supported by National Institutes of Health Grants R29CA66723 and UO-1 CA85078 to BC.

Address reprint requests to: Dr. Bogdan Czerniak, University of Texas, M.D. Anderson Cancer Center, Department of Pathology, Box 085, 1515 Holcombe Boulevard, Houston, Texas 77030-4009. E-mail: bczernia@ mdanderson.org mucosal surface of the bladder can be examined by cystoscopy and biopsies with minimal risk for the patient, and exfoliated urothelial cells can be repeatedly tested for various alterations in voided urine at no risk at all (Gazdar and Czerniak, 2001). Moreover, the simple anatomy and appropriate size of the bladder permit the histologic and genetic mapping studies of invasive cancer and preneoplastic lesions in the entire mucosa of cystectomy specimens. The whole-organ histologic and genetic mapping combined with powerful statistical algorithms, such as the nearest neighbor and binomial maximum likelihood analyses, was previously shown to be efficient in identifying early genetic hits associated with clonal expansion of urothelial cells in preinvasive phases of bladder neoplasia (Chaturvedi et al, 1997; Czerniak et al, 1999, 2000).

Here, we report the results of our studies of the development and evolution of losses of genetic material on chromosome 5 in relation to the progression of bladder neoplasia from preneoplastic conditions to invasive cancer, and we relate the deleted regions on chromosome 5 defined by hypervariable markers to more accurate human genome sequence-based maps. Allelic losses on chromosome 5 were studied in multiple DNA samples from cystectomy specimens with invasive bladder cancer and microscopically recognizable preneoplastic changes in adjacent urothe- 
lium. The markers with statistically significant alterations related to progression of neoplasia from intraurothelial lesions to invasive carcinoma were tested on multiple tumor and voided urine samples of patients with bladder cancer corresponding to different pathogenetic subsets, grades, and stages of the disease. Using this approach we have identified four distinct regions of chromosome 5 that were involved in preinvasive phases of bladder neoplasia and documented that a 9 centimorgan (cM) deleted region mapping to q22-q31.1 exhibited allelic losses in a high proportion of bladder tumors regardless of their pathogenetic subset, grade, and stage. Moreover, allelic losses in this region could be also detected in a high proportion of voided urine samples of patients with bladder cancer. Finally, the deleted regions defined by hypervariable markers were related to more accurate physical maps and bacterial artificial chromosome (BAC) contigs, which enabled the analysis of rapidly emerging human genome sequence databases. The genome sequence analysis of deleted regions provided data on the positions of 138 known genes and revealed several smaller expressed sequence tags (EST) and gene-rich areas as putative targets for further mapping of pathogenetically relevant genes.

\section{Results}

\section{Whole-Organ Histologic and Genetic Mapping}

The initial testing of paired normal and tumor DNA samples from the same patient identified loss of heterozygosity ( $\mathrm{LOH}$ ) in 12 of 38 hypervariable markers. No expansion or shortening of repetitive sequences was identified. None of the cystectomy specimens showed evidence of continuous allelic losses involving large portions of chromosome 5 or complete loss of the entire chromosome, precluding precise mapping of smaller regions. The list of tested markers, their alterations, and chromosomal locations is provided in Figure 1.

Testing of markers with $\mathrm{LOH}$ on multiple mucosal samples of the same cystectomy specimen always revealed a loss of the same allele, suggesting a clonal relationship among cells from individual mucosal samples (Fig. 2). By superimposing distributions of $\mathrm{LOH}$ in individual loci over the histologic maps, we identified two basic distribution patterns of $\mathrm{LOH}$ involving bladder mucosa: scattered and plaque-like (Fig. 2). Some of the plaque-like alterations involved large areas of bladder mucosa with various precursor conditions and even some adjacent areas of morphologically normal urothelium. Such patterns of mucosal involvement implied that $\mathrm{LOH}$ occurred early in the development of urothelial neoplasia, even before microscopically recognizable preneoplastic conditions developed. However, smaller plaques of $\mathrm{LOH}$, restricted to areas of severe dysplasia/carcinoma in situ or invasive cancer, represented late hits associated with progression to the invasive phenotype.

Three-dimensional patterns of $\mathrm{LOH}$ in individual chromosome 5 loci in relation to progression of neo- plasia from precursor conditions to invasive cancer were generated by nearest neighbor analysis. None of the mucosal areas with $\mathrm{LOH}$ was rejected by the nearest neighbor algorithm, indicating that scattered foci of alterations were in fact located within the larger field change in which other regions of chromosome 5 showed LOH.

For a binomial maximum likelihood analysis, the intraurothelial precancerous conditions were classified into two groups: low-grade intraurothelial neoplasia (mild to moderate dysplasia, LGIN) and high-grade intraurothelial neoplasia (severe dysplasia and carcinoma in situ, HGIN). Analysis of logarithm of odds (LOD) scores showed that the markers exhibiting LOH with a statistically significant relationship to the development and progression of urothelial neoplasia were clustered in a large, approximately 70 cM, 5q13.3-q32 region containing several smaller discontinuous areas of allelic losses involving 5q13.3-q22, 5q22-q31.1, and $5 q 31.1-q 32$. The deleted regions, defined by their flanking markers and their predicted size as well as the list of markers within these regions with $\mathrm{LOH}$, are shown in Figure 1. The allelic losses within the region 5q13.3-q22 showed LOH of a marker D5S421 associated with the development of LGIN, which also could be identified in the adjacent areas of microscopically normal urothelium, implicating its involvement in early phases of urothelial neoplasia antecedent to the development of microscopically recognizable preneoplastic conditions. The remaining markers (D5S428, D5S346, and a marker located within the APC gene) mapping to the same region showed $\mathrm{LOH}$ in later phases of urothelial neoplasia associated with the development of HGIN progressing to invasive bladder cancer. The adjacent minimally deleted region within the 5q22-q31.1 involved four markers: MCC, D5S659, D5S2055, and D5S818. The marker D5S659 showed allelic losses associated with the development of LGIN. The three remaining markers mapping to this region developed $\mathrm{LOH}$ in the late phases of urothelial neoplasia, ie, HGIN progressing to invasive carcinoma. An additional smaller region of deletions was found in 5q31.1-q32 and involved markers located within the IRF1 and CSF1R genes. The allelic losses within the CSF1R and IRF1 genes were identified in association with development of LGIN. A separate deleted region mapping to 5 q34 involved marker D5S1465, which revealed LOH in association with the development of HGIN progressing to invasive carcinoma.

\section{Frequency of Allelic Losses on Chromosome 5 in Bladder Tumors and Voided Urine Samples}

Markers showing LOH with a statistically significant relationship to the progression of urinary neoplasia, which clustered in four distinct chromosomal regions including their nearest nonaltered flanking markers, were tested on 37 tumors and 29 voided urine samples of patients with bladder cancer and paired nontumor DNA from peripheral blood lymphocytes (Table 1). $\mathrm{LOH}$ of at least one marker could be identified in 
A.

\section{Chromosome 5}

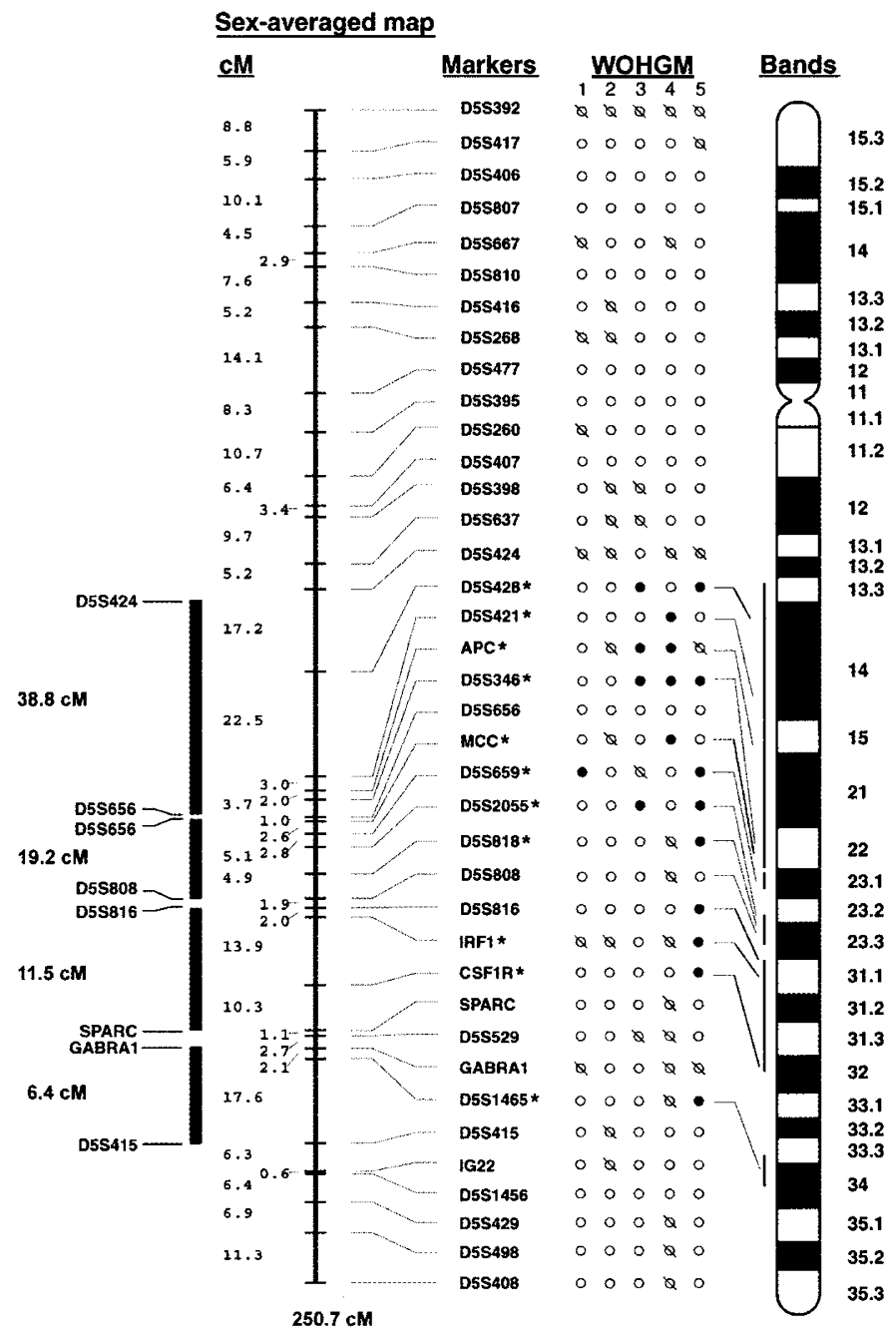

B.

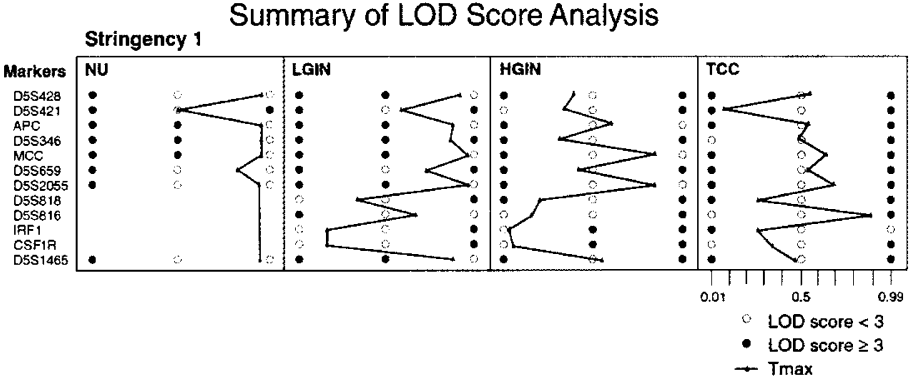

Figure 1.

Whole-organ histologic and genetic mapping of deleted regions on chromosome 5 involved in progression of human bladder neoplasia from preneoplastic intraurothelial lesion to invasive cancer. A, Genetic map of chromosome 5 with a list of tested markers and their distances. Chromosomal locations are provided for altered markers only. All the markers were positioned on the map according to the Cooperative Human Linkage Center map (version 4.0). Asterisks on the right side of the markers indicate statistically significant association between an altered marker and urothelial neoplasia as established by logarithm of odds (LOD) scores. Bars on the left side of the chromosomal vector identify the deleted regions associated with the development and progression of urothelial neoplasia. The regions of allelic losses defined by the nearest nonaltered flanking markers and their predicted size in centimorgans (cM) are as follows: 5q13.3-q22 (D5S424-D5S656, 38.8 cM), 5q22-q31.1 (D5S656-D5S808, 19.2 cM), 5q31.1-q32 (D5S816-SPARC, $11.5 \mathrm{cM}$ ), and 5q34 (GABRA1-D5S415, $6.4 \mathrm{cM})$. The relationship of markers with loss of heterozygosity (LOH) to various phases of neoplasia is provided in the LOD score table shown in B. (cM, centimorgans; WOHGM, whole-organ histologic and genetic mapping of individual cystectomy specimens consecutively numbered 1 through $5 . \bigcirc$, nonaltered marker; $\bullet$, markers with $\mathrm{LOH} ; \varnothing$, noninformative marker.) B, Summary of binomial maximum likelihood analysis testing the relationship among LOH in individual chromosome 5 loci and progression of urothelial neoplasia from in situ precursor conditions to invasive transitional cell carcinoma (TCC). Cumulative LOD scores for markers with LOH were calculated at variable $\theta=(0.01,0.5$, and 0.99$)$ and tested against Tmax. The significance of allelic losses in individual loci was analyzed for normal urothelium (NU), low-grade intraurothelial neoplasia (LGIN), high-grade intraurothelial neoplasia (HGIN), and TCC. To simplify the data, stringency 1 calculations are presented only. The patterns of significant LOD scores are as described in "Materials and Methods." Note that significant patterns of LOD scores typically parallel the high Tmax values. $(\bigcirc$, LOD score $<3$;, LOD score $\geq 3$.) 
A.

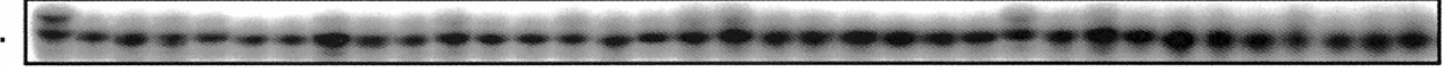

$\begin{array}{lllllllllllllllllllllllllllllllllllllll}.7 & .3 & .3 & .4 & .3 & .2 & .3 & .3 & .3 & .4 & .4 & .3 & .3 & .4 & .4 & .4 & .6 & .6 & .5 & .4 & .3 & .3 & .3 & .3 & .7 & .4 & .4 & .3 & .2 & .4 & .4 & .6 & .4 & .6 & .6\end{array}$

B.

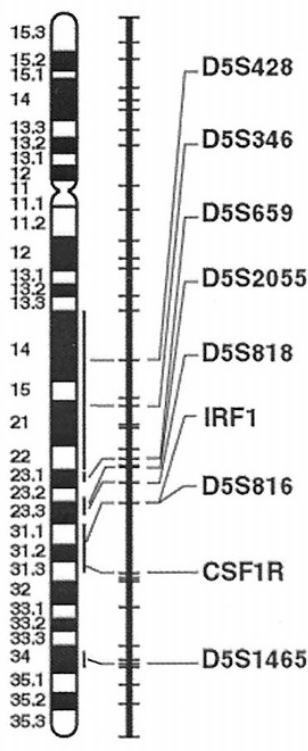

C.

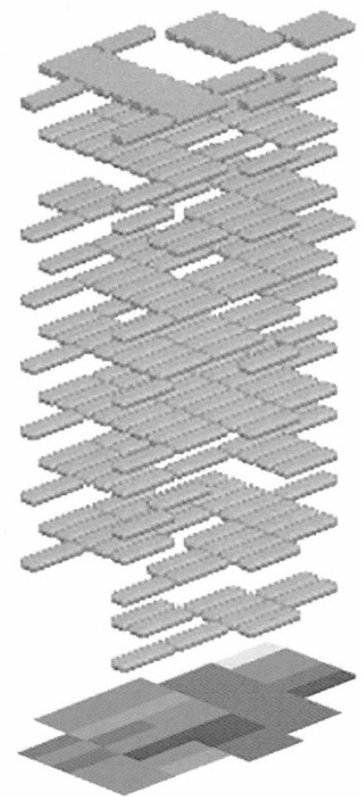

Histologic map

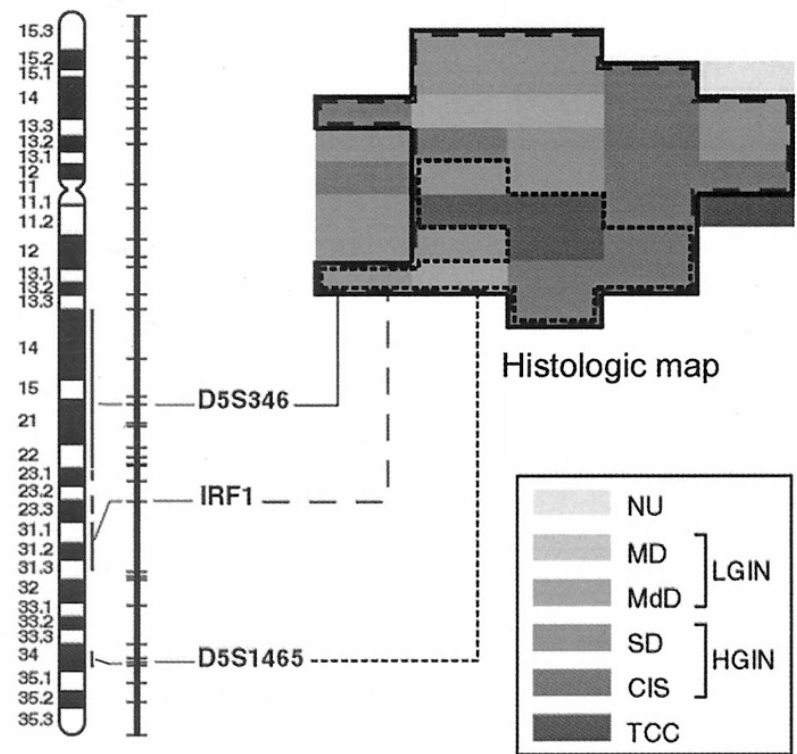

Figure 2.

Assembly of whole-organ histologic and genetic maps. A, An example of a marker tested on multiple mucosal samples from the cystectomy specimen (Map 5). Marker IRF1 shows LOH in samples corresponding to TCC (Samples 4, 15, and 16) as well as in samples exhibiting changes consistent with LGIN (Samples 13, 19, 22, and 27) and HGIN (Samples 2, 3, 5-12, 14, 17, 18, 20, 21, 23, 24, 26, 28-35). Sample 1 represents allelic patterns of the same marker from peripheral blood of the same patient and serves as a control. The presence of $\mathrm{LOH}$ in all samples was confirmed by densitometry and is expressed as an OD ratio below each sample. OD $<0.5$ is indicative of LOH. B, Example of chromosome 5 allelic losses in a single cystectomy specimen (Map 5) with invasive nonpapillary urothelial carcinoma assembled by nearest neighbor analysis. The vertical axis represents a chromosome 5 map with positions of markers and their chromosomal locations. Only altered markers are shown. The shaded blocks represent areas of urinary bladder mucosa with $\mathrm{LOH}$ as they relate to progression of neoplasia presented by a histologic map of cystectomy in the background. Note that several markers, including IRF1, show LOH in a form of a plaque involving a large area of urinary bladder mucosa. The code for a histologic map is shown in C. C, Examples of LOH distributions superimposed on a histologic map of cystectomy specimen (Map 5). Markers D5S346 and IRF1 show a plaque-like LOH involving almost the entire urinary bladder mucosa. Marker D5S1465 shows LOH involving a smaller area of urinary bladder mucosa located within a larger plaque of LOH that involved markers D5S346 and IRF1. Open boxes delineated by lines indicate areas of urinary bladder mucosa with alterations in a given locus. The background-shadowed area represents a histologic map of cystectomy specimen depicting distribution of various intraurothelial precursor conditions and TCC. Histologic map code: NU, normal urothelium; MD, mild dysplasia; MdD, moderate dysplasia; SD, severe dysplasia; CIS, carcinoma in situ; TCC, invasive transitional cell carcinoma.

$38.4 \%$ of informative tumors and $58.6 \%$ of informative voided urine samples. The highest frequency of $\mathrm{LOH}$ in both tumor and voided urine samples was found in region mapping to 5 q22-q31.1 and could be identified in $27.0 \%$ and $27.5 \%$ of cases, respectively. The second most frequently deleted region mapping to 5q13.3-q22 showed $\mathrm{LOH}$ in approximately $24 \%$ of tumor and voided urine samples. In two remaining loci mapping to 5q31.1-q32 and 5q34, the allelic losses in both tumor and voided urine samples could be identified in $18 \%$ or fewer of the cases. The statistical analysis of frequency of $\mathrm{LOH}$ in individual loci and minimally deleted regions on chromosome 5 have shown that none of the $\mathrm{LOH}$ could be related to specific pathogenetic subsets, histologic grade, or stage of the tumor (data not shown). Although, the allelic losses within 5q13.3-q22 were the most fre- quent, the markers with LOH mapping to this area did not form a distinct narrow region of allelic losses. On the other hand, the two neighbor markers, D5S2055 and D5S818, mapping to 5q22-q31.1 defined a distinct region of allelic losses that could be identified in $21.6 \%$ and $27.5 \%$ of bladder tumor and urine samples, respectively. Thus, the minimally deleted region flanked by markers D5S659 and D5S808, spanning approximately $9 \mathrm{cM}$, may contain tumor suppressor genes with important roles in urinary bladder carcinogenesis.

\section{Analysis of Contigs and Genome Sequence Databases Spanning the Deleted Regions}

The analysis of human genome contig and sequencing databases spanning the deleted regions on chromo- 
Table 1. Frequency of Allelic Losses at Different Regions on Chromosome 5 in Bladder TCC and Voided Urine Samples ${ }^{a}$

\begin{tabular}{|c|c|c|c|c|c|}
\hline \multirow{3}{*}{$\begin{array}{l}\text { Deleted } \\
\text { regions }\end{array}$} & \multirow[b]{3}{*}{ Markers } & \multicolumn{4}{|c|}{ Frequency of $\mathrm{LOH}(\%)$} \\
\hline & & \multicolumn{2}{|c|}{$\begin{array}{l}\text { Bladder tumor samples } \\
\qquad(\mathrm{n}=37)\end{array}$} & \multicolumn{2}{|c|}{$\begin{array}{l}\text { Voided urine samples } \\
\qquad(\mathrm{n}=29)\end{array}$} \\
\hline & & Marker & Region & Marker & Region \\
\hline \multirow[t]{4}{*}{$5 q 13.3-q 22$} & D5S428 & 19.4 & 24.3 & 13.6 & 24.1 \\
\hline & D5S421 & 2.8 & & 12.0 & \\
\hline & APC & 5.9 & & 7.7 & \\
\hline & D5S346 & 19.4 & & 7.7 & \\
\hline \multirow[t]{4}{*}{$5 q 22-q 31.1^{b}$} & MCC & 5.6 & 27.0 & 0.0 & 27.5 \\
\hline & D5S659 & 9.1 & & 0.0 & \\
\hline & D5S2055 & 11.1 & 21.6 & 16 & 27.5 \\
\hline & D5S818 & 16.7 l & & $17.9 \mid$ & \\
\hline \multirow[t]{2}{*}{$5 q 31.1-q 32$} & IRF1 & 8.3 & 8.1 & 12.0 & 17.2 \\
\hline & CSF1R & 2.7 & & 11.5 & \\
\hline $5 q 34$ & D5S1465 & 12.5 & 12.5 & 4.0 & 4.0 \\
\hline
\end{tabular}

TCC, transitional cell carcinoma; LOH, loss of heterozygosity.

${ }^{a}$ Raw data used for this analysis can be obtained from our web site (http://www.mdanderson.org/Departments/GenomeMaps/)

${ }^{b}$ The distinct region of allelic losses mapping to 5q22-q31.1 defined by the two neighbor markers (D5S2055 and D5S818) is identified by a solid vertical bar followed by a combined \% of $\mathrm{LOH}$ for these markers.

some 5 is summarized in Figure 3. The four deleted regions on chromosome 5 contain 138 known genes. In addition, multiple EST were assigned to individual deleted regions identifying several smaller gene-rich areas. The most frequently deleted region mapping to $5 \mathrm{q} 22-\mathrm{q} 31.1$ contained areas with high densities of EST and known genes, some of them with putative tumor suppressor activities, further supporting a concept of its potential pathogenetic relevance for bladder carcinogenesis.

\section{Discussion}

The strategy of whole-organ histologic and genetic mapping permitted us to analyze the relationships between genetic alterations in continuity with disease progression from morphologically normal urothelium through microscopically recognizable in situ precursor lesions to invasive cancer throughout the entire cystectomy specimen. Using this approach, we identified a large 5q13.3-q32 region of allelic losses that contained several smaller discontinuous deleted areas mapping to 5q13.3-q22, 5q22-q31.1, and 5q31.1-q32. It turned out that regions mapping to $5 q 13.3-q 22$ and q22-q31.1 were most frequently altered and could be identified in approximately $24 \%$ of bladder tumors and $27 \%$ voided urine samples. The two neighbor markers (D5S2055 and D5S818) mapping to 5q22-q31.1 define a distinct $9 \mathrm{cM}$ region involved in intraurothelial phases of bladder neoplasia and could be identified in a significant proportion of bladder tumors and voided urine samples of patients with bladder cancer. Thus, this region may contain genes that play an important role in early phases of urothelial neoplasia and its progression to invasive disease.

To provide more detailed information on the chromosome 5 target loci involved in bladder carcinogenesis, we compared the positions of markers defining the deleted regions with those used to generate the most recent version of GeneMap'99. We hoped to find the new positions for markers based on physical map data and identify the nearest substitutes for those markers that were not present on the radiation hybrid panel-based physical map produced by the International Radiation Hybrid Mapping Consortium. The resulting computational analysis of the DNA sequences in the target regions allowed us to identify several smaller areas with high known gene and EST densities, which provided additional clues to potential pathogenetic relevance of the loci for bladder carcinogenesis. It should be noted that these data represent a snapshot of genome resources available at the moment. The rapidly expanding human genome sequence-based databases and their attendant informatic resources will necessitate a constant redrawing of the picture presented in this communication.

The original histologic mapping studies of cystectomy specimens containing bladder cancer and intraurothelial precursor conditions in adjacent mucosa were performed approximately two decades ago. These studies provided the foundation for the generally accepted dual-track concept of urinary bladder carcinogenesis. It postulates that bladder tumors develop via two distinct pathways, one leading to the development of papillary tumors and the other to nonpapillary tumors (Czerniak and Herz, 1995; Koss et al, 1974). Nearly $80 \%$ of bladder tumors are superficial papillary lesions that originate from flat hyperplastic lesions. In contrast, invasive nonpapillary bladder cancers typically present in a patient without a history of superficial lesions and represent a progression of severe dysplasia or flat transitional carcinoma in situ. The distinct pathogenesis of papillary and nonpapillary bladder tumors has been addressed more recently in several molecular studies (Simoneau and Jones, 1994; 


\section{Chromosome 5}

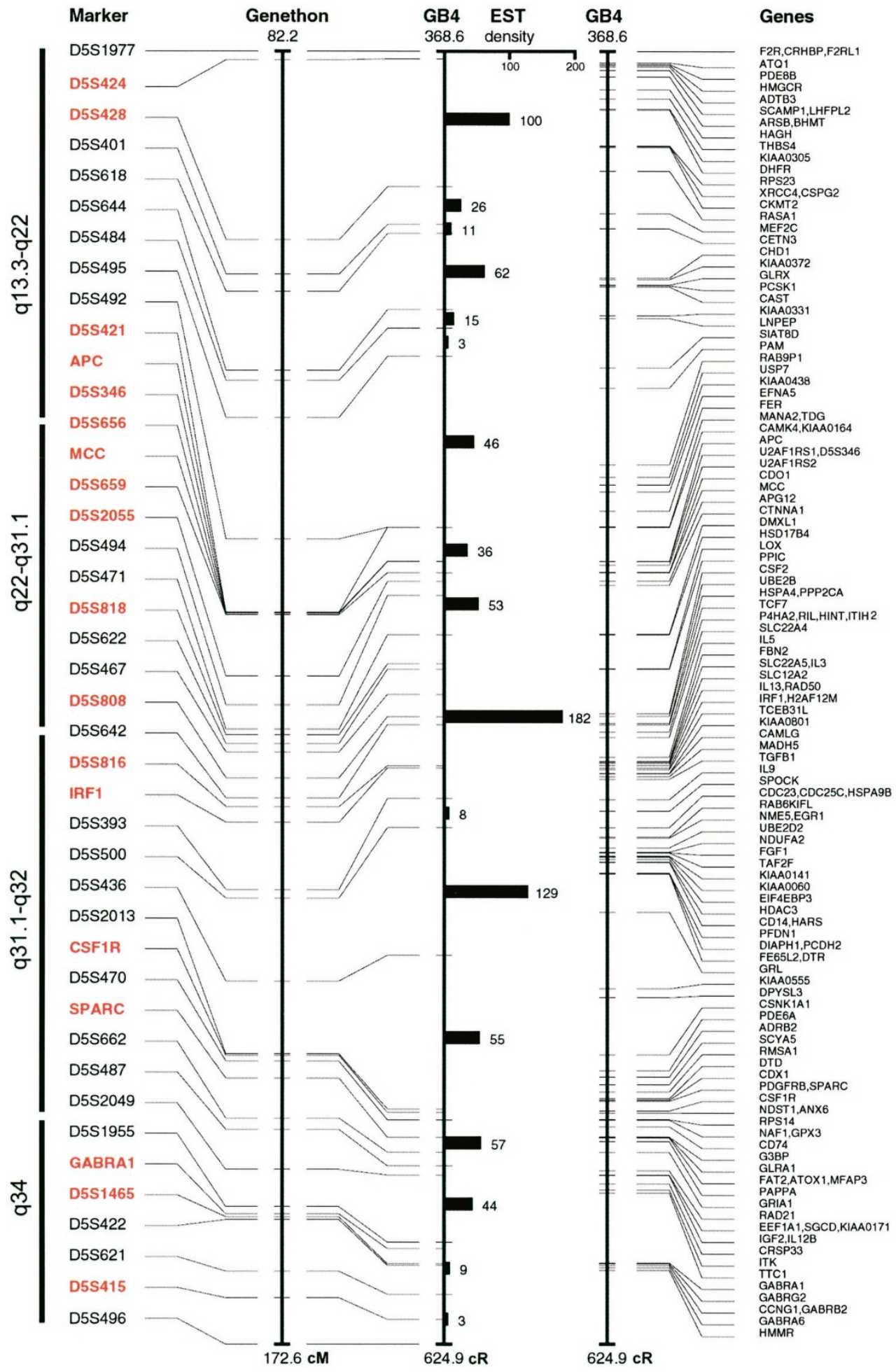

Figure 3.

Summary of physical map analysis spanning the deleted regions of chromosome 5 performed as described in "Material and Methods." Original markers and substitutes for markers with LOH based on the closest proximity were placed on the Genethon map and were repositioned on the GB4 radiation hybrid panel-based physical map. The new positions for the Genethon markers with $\mathrm{LOH}$, as well as flanking markers on the GB4 map, were identified by electronic PCR search of bacterial artificial chromosome (BAC) contigs. In addition, multiple alternative markers based on their proximity to markers with LOH were identified and added to the map. The original Genethon markers with $\mathrm{LOH}$ are shown in red. All other substitute and flanking markers are printed in black. In addition, average expressed sequence $\operatorname{tag}$ (EST) density for regions flanked by individual markers placed on the GB4 map and a list of 138 known genes mapping to the target regions are shown. To simplify the diagram, contig data used for this analysis are not provided. More complete data with alternative positions of the genes can be obtained from our Web site: http://www.mdanderson.org/bladdergenomicmaps. (cM, centimorgan; cR, centiray) 
Spruck et al, 1994). It appears that the low-grade, superficially growing, papillary tumors exhibit fewer chromosomal and molecular abnormalities than highgrade invasive carcinomas (Dalbagni et al, 1993; Simon et al, 1998). Typically, they are characterized by trisomies of chromosomes 1 and 7 and monosomies of chromosome 9. High-grade nonpapillary tumors however, develop multiple chromosomal rearrangements, deletions, and amplifications involving chromosomes 3, 11, 13, 17, 18, and 22 (Czerniak et al, 2000; Rosin et al, 1995; Takahashi et al, 1998). These tumors also frequently show alterations of major tumor suppressor genes such as p53 and Rb, as well as the genes of their regulatory pathways.

Several putative tumor suppressor gene loci mapping to chromosome 5 have been implicated in the development of common epithelial human malignancies, including urinary bladder cancer (Bohm et al, 1997; Gorgoulis et al, 2000; Hejlik et al, 1997; Luft et al, 1999; Nozawa et al, 1998; Onuki et al, 1999; Saran et al, 1996). Early karyotyping studies identified deletions and amplifications of both arms of chromosome 5 , suggesting the involvement of several genes in human bladder carcinogenesis (Atkin and Fox, 1990; Gibas et al, 1986; Klingelhutz et al, 1991). More recent studies using comparative genomic hybridization and hypervariable markers have identified three distinct regions on chromosome 5 as being involved in the progression of urinary bladder cancer (Bohm et al, 1997; Koo et al, 1999; von Knobloch et al, 2000; Voorter et al, 1995). An area mapping to chromosome $5 p$ between markers D5S1473 and D5S819 was commonly amplified, and two distinct allelic regions were deleted in 5q22-q23 and 5q33-q34 (von Knobloch et al, 2000).

Our current studies provide evidence that a distinct tumor suppressor gene locus mapping to 5q22-q31.1 may be involved in the progression of bladder neoplasia from precursor conditions to invasive cancer. Given the large size of this locus $(9 \mathrm{cM})$, the task of identifying pathogenetically relevant genes in this area is extremely complex, time consuming, and not always successful if conventional positional cloning strategies are used. However, with the advent of novel BAC-based DNA microarray technologies, rapid mapping of large deleted or amplified regions spanning several cM becomes feasible (Lucito et al, 2000; Pollack et al, 1999). Our data should lead to the identification of novel genes involved in clinically occult preinvasive phases of human bladder carcinogenesis. Such genes could serve as markers for early detection of bladder cancer and be used for risk assessment in population-based studies.

\section{Materials and Methods}

\section{Whole-Organ Histologic and Genetic Mapping}

Radical cystectomy specimens from five patients with previously untreated sporadic high-grade invasive transitional cell carcinoma (TCC) of the bladder were used for the whole-organ histologic and genetic map- ping as previously described (Chaturvedi et al, 1997; Czerniak et al, 1999, 2000). All patients were men, and their ages ranged from 47 to 78 years (mean $=66.4 \pm$ 11.9 years SD).

In brief, each fresh cystectomy specimen was opened longitudinally along the anterior wall of the bladder and pinned down to a paraffin block. The entire mucosa was than divided into $1 \times 2 \mathrm{~cm}$ rectangular samples and evaluated microscopically on frozen sections. The tissue of interest was microdissected from the frozen block and used to prepare a urothelial cell suspension by mechanically scraping the urothelial mucosa or gently shaking invasive tumor samples. Only those specimens that yielded more than $90 \%$ of microscopically recognizable intact urothelial or tumor cells in each sample were accepted for the study and used for DNA extraction. This procedure provided $49,39,65,42$, and 39 DNA samples from each cystectomy specimen that corresponded to microscopically identified intraurothelial precursor conditions or invasive carcinoma. As a control, DNA extracted from the peripheral blood lymphocytes and/or from normal tissue in the resected specimen of each patient was used.

The intraurothelial precancerous changes were classified as mild, moderate, and severe dysplasia or carcinoma in situ. The tumors were classified according to the three-tier histologic grading system of the World Health Organization (Mostofi, 1999). The growth pattern of papillary versus nonpapillary or solid tumors and the depth of invasion were also recorded. In four of the five cystectomy specimens, a single focus of Grade 3 nonpapillary urothelial carcinoma invaded the muscularis propria and was accompanied by extensive precancerous lesions ranging from mild dysplasia to carcinoma in situ. In the remaining case, multiple foci of carcinoma were present. One focus represented a Grade 3 nonpapillary urothelial carcinoma with transmural invasion of the bladder wall and involvement of perivesical adipose tissue. Two additional foci of carcinoma represented Grade 3 papillary urothelial carcinoma without invasion. Like the other four cases, this case exhibited changes ranging from mild dysplasia to carcinoma in situ over extensive areas of the urinary bladder mucosa. The results of microscopic evaluation of individual samples from five cystectomy specimens were recorded and stored in a computer database as histologic maps.

\section{Tumors and Voided Urine Samples of Patients with Bladder Cancer}

The markers of chromosome 5 that were identified as significantly altered by the whole histologic and genetic mapping were tested in 37 tumor and 29 voided urine samples. The tumors were classified according to the three-tier histologic grading system of the World Health Organization (Mostofi, 1999). The growth pattern, tumor grade, and depth of invasion were also recorded. Levels of invasion were recorded according to the tumor node metastasis (TNM) staging system (Sobin and Wittekind, 1997). DNA was extracted from 
individual bladder tumors and sediments of voided urine samples as previously described (Chaturvedi et al, 1997). For controls, DNA was also extracted from the peripheral blood lymphocytes and/or normal tissue in the resected specimens from each patient.

\section{Microsatellites}

A set of primers for 38 microsatellite loci on chromosome 5 based on the integrated sex-averaged microsatellite map from Genethon (version: March 1966) and updated by Cooperative Human Linkage Center (version 4.0) was obtained from Research Genetics (Huntsville, Alabama). The markers selected for testing exhibited high levels of heterozygosity and uniform distribution covering all regions of chromosome 5 . Figure 1 lists hypervariable markers and their positions on chromosome 5. The allelic patterns of markers were resolved on $6 \%$ polyacrylamide gels after their amplification using polymerase chain reaction as previously described (Chaturvedi et al, 1997). A minimum $50 \%$ reduction in signal intensity was required to be considered as evidence of LOH. Tests with questionable results were repeated. In such cases, the densitometric measurements were performed to ensure objective reading of the data. Testing of markers was performed in two steps. Initially, all markers were tested on paired normal and tumor DNA samples. This revealed $\mathrm{LOH}$ in 12 markers, which were tested on all mucosal DNA samples by whole-organ histologic and genetic mapping.

\section{Analysis of LOH Data}

The data were organized and analyzed as previously described (Chaturvedi et al, 1997; Czerniak et al, 1999, 2000). In brief, the information on $\mathrm{LOH}$ in individual loci was entered into the data files and superimposed over the histologic maps. Initial data consisted of chromosomal vectors with a list of $\mathrm{LOH}$ in individual loci and coordinates for locations of mucosal samples, which could be used to plot the distribution of $\mathrm{LOH}$ to microscopically classified urothelial changes. By superimposing plots of $\mathrm{LOH}$ over the histologic maps, we identified the areas of bladder mucosa with altered markers and analyzed their relationship to intraurothelial precursor conditions and invasive cancer. Three-dimensional displays of LOH in relation to the progression of neoplasia from precursor intraurothelial conditions to invasive cancer were generated and initially analyzed by the nearest neighbor algorithm (Hartigan, 1975).

The relationship between altered markers and the progression of urothelial neoplasia from precursor conditions to invasive cancer was tested by a binomial maximum likelihood analysis, and the significance of the relationship was expressed as a LOD score (Ott, 1991). We chose LOD scores because they represent a powerful method of likelihood analysis that can verify the statistical significance of the relationship among patterns of sequential events. The LOD scores were applied in their generic mathematical sense as likelihood tests of events, not as in their common use to test the linkage in familial disorders with meiotic segregation of the phenotype at a recombination fraction $\theta=0.5$. In sporadic cancer, when microscopically defined stages of cancer progression are used as standards of sequential events and there is a mitotic transmission of the phenotype, the null hypothesis is more appropriately verified at a recombination factor $\theta$ differing from 0.5 . Hence, cumulated LOD scores were calculated at variable $\theta=0.01,0.5$, and 0.99 . A pattern of LOD scores $\geq 3$ at $\theta=0.01$ or $\theta=$ 0.99 , and LOD scores $<3$ at $\theta=0.5$ for the same marker, was considered significant. The strongest association between altered marker and neoplasia was when a LOD score was $\geq 3$ and $\theta=0.99$ and 0.5 , and $<3$ at $\theta=0.01$. Stringency 1 designated LOD scores for specific stages of neoplasia. Stringency 2 designated LOD scores for progression to higher stages of neoplasia. The analysis of the relationship among $\mathrm{LOH}$ in individual loci and various clinicopathological parameters of tumors and of voided urine samples was tested by Gehan's generalized Wilcoxon, and log-rank tests $(p \leq 0.05$ was considered significant).

\section{Analysis of Contigs and Human Genome Sequence Databases Spanning the Deleted Regions}

The initial resource available for the whole-organ histologic and genetic mapping of deleted regions on chromosome 5 consisted of a list of hypervariable markers based on integrated sex-averaged microsatellite maps from Genethon and the Cooperative Human Linkage Center. However, human genome sequence-based databases with more accurate physical maps become available during our studies. Thus, to relate our data to sequence maps of the human genome, we initially looked for overlap between the original sets of markers which defined the deleted regions and those used to generate the current version of GeneMap'99 (http://www.ncbi.nlm.nih.gov/ genemap99/). This resource represents the most complete melding of the microsatellite-based genetic map data from Genethon (http://www.genethon.fr/) with the GeneBridge 4 (GB4) and Stanford G3 radiation hybrid panel-based physical map produced by the International Radiation Hybrid Mapping Consortium (http://www.ncbi.nlm.nih.gov/genemap99/page.cgi?F =Consortium.html).

Although some of the original Marshfield sexaveraged markers defining the deleted regions can be found in GeneMap'99, substitutes for those not found were proposed primarily because of their physical proximity. The resources used for these substitutions included the "Golden Path" Genome Browser (http:// genome.ucsc.edu/), containing the whole-genome fingerprint map from Washington University (http:// genome.wustl.edu/gsc/human/human_database.shtml), the sequence-based mapping tools at the Ensembl website produced at the European Bioinformatics Institute (http://www.ensembl.org/), and the integrated MapViewer browser from the National Center for Biotechnol- 
ogy Information (NCBI) (http://www.ncbi.nlm.nih.gov/ cgi-bin/Entrez/hum_srch?chr=hum_chr.inf\&query). These same resources, together with NCBI's LocusLink (http://www.ncbi.nlm.nih.gov/LocusLink), were used to scan the marker-defined deleted regions for both known genes and EST clusters based on Unigene (http:// www.ncbi.nlm.nih.gov/UniGene/Hs.Home.html). The Baylor College of Medicine Search Launcher (http:// www.hgsc.bcm.tmc.edu/SearchLauncher/) provided the portal and integration for these links.

After reorientation of contigs and sequence databases, multiple electronic PCR searches were performed to find and relocate the original set of markers on the GB4 and sequence maps. As a general rule, we attempted to locate the original markers and substitute GB4 markers within a single BAC clone. Because, in the majority of instances, complete continuous sequences of BAC clones were not available yet, it was impossible to find the exact order of paired original and substitute markers within the target BAC clone. When the original and substitute markers were not located within the same BAC clone, the most proximal substitute markers within the contigs spanning the analyzed regions were provided.

\section{References}

Atkin NB and Fox MF (1990). $5 q$ deletion. The sole chromosome change in a carcinoma of the bladder. Cancer Genet Cytogenet 46:129-131.

Bohm M, Kirch H, Otto T, Rubben H, and Wieland I (1997). Deletion analysis at the DEL-27, APC, and MTS1 loci in bladder cancer: LOH at the DEL-27 locus on $5 \mathrm{p} 13-12$ is a prognostic marker of tumor progression. Int $\mathrm{J}$ Cancer 74 : 291-295.

Chaturvedi V, Li L, Hodges S, Johnston D, Ro JY, Logothetis C, von Eschenbach AC, Batsakis JG, and Czerniak B (1997). Superimposed histologic and genetic mapping of chromosome 17 alterations in human urinary bladder neoplasia. Oncogene 14:2059-2070.

Czerniak B, Chaturvedi V, Li L, Hodges S, Johnston D, Ro JY, Luthra R, Logothetis C, von Eschenbach AC, Grossman B, Benedict WF, and Batsakis JG (1999). Superimposed histologic and genetic mapping of chromosome 9 in progression of human urinary bladder neoplasia: Implications for a genetic model of multistep urothelial carcinogenesis and early detection of urinary bladder cancer. Oncogene 18:11851196.

Czerniak B and Herz F (1995). Molecular biology of urinary bladder cancer. In: Koss LG, editor. Diagnostic cytology of the urinary tract with histopathologic and clinical correlations. Philadelphia: Lippincott-Raven, 345-359.

Czerniak B, Li L, Chaturvedi V, Ro JY, Johnston DA, Hodges S, and Benedict WF (2000). Genetic modeling of human urinary bladder carcinogenesis. Genes Chromosomes Cancer 27:392-402.

Dalbagni G, Presti J, Reuter V, Fair WR, and Cordon-Cardo C (1993). Genetic alterations in bladder cancer. Lancet 342: 469-471.

Gazdar AF and Czerniak B (2001). Filling the void-urinary markers for bladder cancer risk and diagnosis. J Natl Cancer Inst 93:413-414.
Gibas Z, Prout GR, Pontes JE, Connolly JG, and Sandberg AA (1986). A possible specific chromosome change in transitional cell carcinoma of the bladder. Cancer Genet Cytogenet 19:229-238.

Gorgoulis VG, Mariatos G, Manolis EN, Zacharatos P, Kotsinas A, Liloglou T, Vogiatzi T, Tsagkaraki A, Kokotas S, Tsoli E, Alchanatis M, Sfikakis PP, Asimacopoulos PJ, Field JK, and Kittas C (2000). Allelic imbalance at the $5 q 14$ locus is associated with decreased apoptotic rate in non-small cell lung carcinomas (NSCLCs). Possible synergistic effect with p53 gene alterations on apoptosis. Lung Cancer 28:211-224.

Greenlee RT, Murray T, Bolden S, and Wingo PA (2000). Cancer Statistics 2000. CA Cancer J Clin 50:7-33.

Hartigan JA (1975). Clustering algorithms. New York: John Wiley \& Sons, Inc.

Hejlik DP, Kottickal LV, Liang H, Fairman J, Davis T, Janecki T, Sexton D, Perry W 3rd, Tavtigian SV, Teng DH, and Nagarajan L (1997). Localization of SMAD5 and its evaluation as a candidate myeloid tumor suppressor. Cancer Res 57: 3779-3783.

Klingelhutz AJ, Wu SQ, and Reznikoff CA (1991). Nonrandom chromosome losses in tumorigenic revertants of hybrids between isogeneic immortal and neoplastic human uroepithelial cells. Somat Cell Mol Genet 17:551-565.

Koo SH, Kwon KC, Ihm CH, Jeon YM, Park JW, and Sul CK (1999). Detection of genetic alterations in bladder tumors by comparative genomic hybridization and cytogenetic analysis. Cancer Genet Cytogenet 110:87-93.

Koss LG, Tiamson EM, and Robbins MA (1974). Mapping cancerous and precancerous bladder changes. A study of the urothelium in ten surgically removed bladders. JAMA 227:281-286.

Lucito R, West J, Reiner A, Alexander J, Esposito D, Mishra B, Powers S, Norton L, and Wigler M (2000). Detecting gene copy number fluctuations in tumor cells by microarray analysis of genomic representations. Genome Res 10:17261736.

Luft F, Gebert J, Schneider A, Melsheimer P, and von Knebel Doeberitz M (1999). Frequent allelic imbalance of tumor suppressor gene loci in cervical dysplasia. Int $\mathrm{J}$ Gynecol Pathol 18:374-380.

Mostofi FK, editor (1999). Histological typing of urinary bladder tumours. New York: Springer.

Nozawa H, Oda E, Ueda S, Tamura G, Maesawa C, Muto T, Taniguchi T, and Tanaka N (1998). Functionally inactivating point mutation in the tumor-suppressor IRF-1 gene identified in human gastric cancer. Int J Cancer 77:522-527.

Onuki N, Wistuba II, Travis WD, Virmani AK, Yashima K, Brambilla E, Hasleton P, and Gazdar AF (1999). Genetic changes in the spectrum of neuroendocrine lung tumors. Cancer 85:600-607.

Ott J (1991). Analysis of human genetic linkage. Baltimore: Johns Hopkins University Press.

Pollack JR, Perou CM, Alizadet AA, Eisen MB, Pergamenschikov A, Williams CF, Jeffrey SS, Botstein D, and Brown PO (1999). Genome-wide analysis of DNA copy number changes using cDNA microarrays. Nat Genet 20:41-46.

Rosin MP, Cairns P, Epstein JI, Schoenberg MP, and Sidransky D (1995). Partial allelotype of carcinoma in situ of the human bladder. Cancer Res 55:5213-5216. 
Saran KK, Gould D, Godec CJ, and Verma RS (1996). Genetics of bladder cancer. J Mol Med 74:441-415.

Simon R, Burger H, Brinkschmidt C, Bocker W, Hertle L, and Terpe HJ (1998). Chromosomal aberrations associated with invasion in papillary superficial bladder cancer. $J$ Pathol 185:345-351.

Simoneau AR and Jones PA (1994). Bladder cancer: The molecular progression to invasive disease. World $\mathrm{J}$ Urol 12:89-95.

Sobin LH and Wittekind C, editors (1997). TNM classification of malignant tumors, 5th ed. New York: Wiley-Liss.

Spruck CH, Ohneseit PF, Gonzalez-Zulueta M, Esrig D, Miyao N, Tsai YC, Lerner SP, Schmutte C, Yang AS, and Cote R, (1994). Two molecular pathways to transitional cell carcinoma of the bladder. Cancer Res 54:784-788.
Takahashi T, Habuchi T, Kakehi Y, Mitsumori K, Akao T, Terachi T, and Yoshida O (1998). Clonal and chronological genetic analysis of multifocal cancers of the bladder and upper urinary tract. Cancer Res 58:5835-5841.

von Knobloch R, Bugert P, Jauch A, Kalble T, and Kovacs G (2000). Allelic changes at multiple regions of chromosome 5 are associated with progression of urinary bladder cancer. J Pathol 190:163-168.

Voorter C, Joos S, Bringuier PP, Vallinga M, Poddighe P, Schalken J, du Manoir S, Ramaekers F, Lichter P, and Hopman A (1995). Detection of chromosomal imbalances in transitional cell carcinoma of the bladder by comparative genomic hybridization. Am J Pathol 146:1341-1354. 